
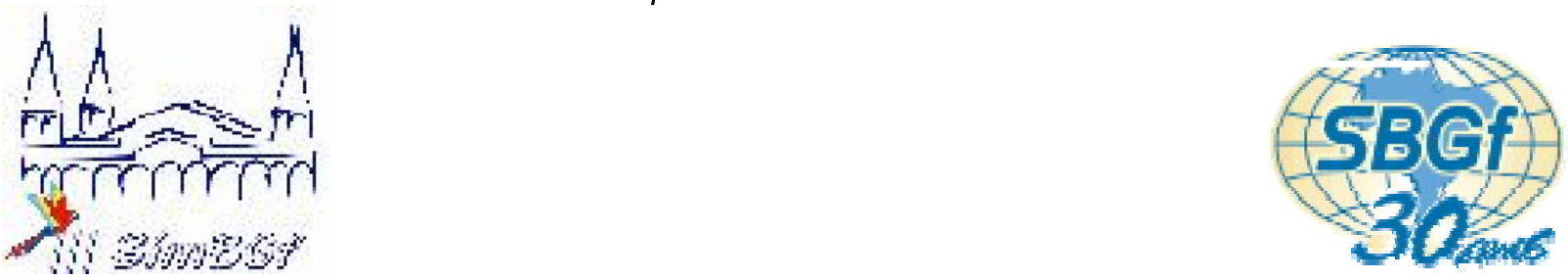

\title{
Modelagem Flexural das Bacias de Santos, Campos e sul do Espírito Santo: Resultados Preliminares
}

Fernando Lessa Pereira ${ }^{1}$, Victor Sacek1 \& Naomi Ussami', 'Departamento de Geofísica, Instituto de Astronomia, Geofísica e Ciências Atmosférica, Universidade de São Paulo (IAG/USP)

Copyright 2008, SBGf - Sociedade Brasileira de Geofísica

Este texto foi preparado para a apresentaçāo no III Simpósio Brasileiro de Geofísica, Belém, 26 a 28 de novembro de 2008. Seu conteúdo foi revisado pelo Comitê Técnico do III SimBGt, mas nāo necessariamente representa a opiniāo da SBGf ou de seus associados. É proibida a reprodução total ou parcial deste material para propósitos comerciais sem prévia autorizaçāo da SBGt.

\section{Abstract}

The distribution of flexural uplift due to post-salt sedimentary loads in Santos, Campos and South Espírito Santo basins was modeled using a 3-D thin-elastic plate model with laterally and temporally variation in effective elastic thickness (Te). The predicted flexural uplifts are calculated since Cenomanian time up to present, divided into 6 main periods. The results show that during the entire period, sedimentation in Santos basin was continuous due to uplift, erosion and Serra do Mar escarpment retreat. On the other hand, erosion and sedimentation in Campos and south Espírito Santos basins are not continuous and might have a source due to uplift and erosion caused by thermo-magmatic events, one within 85-70 Ma and another at 65-28 Ma periods. The present day flexural bulge maximum amplitude extends over Serra do Mar and Serra da Mantiqueira and may be the main cause of geomorphological and topographic features of the SE Brazilian coast.

\section{Introdução}

As bacias de Campos, Santos e Espírito Santo originaram-se no rifteamento da litosfera que levou à abertura do oceano Atlântico Sul (Chang et al., 1992). No processo de desenvolvimento da bacia um componente importante é a subsidência flexural devida à carga sedimentar depositada na região imersa (offshore) que causa uma elevação na porção emersa (onshore) do continente. A distribuição das cargas controla este soerguimento, o qual estará sujeito a ação intempérica funcionando como área fonte para a sedimentação seguinte durante o preenchimento das bacias (Watts \& Ryan, 1976). Uma quantificação dessa subsidência permite estabelecer modelos mais realistas sobre o processo deposicional e conseqüentemente uma melhor previsão sobre a distribuição das rochas fonte, reservatório e retentora de petróleo e gás.

As bacias sedimentares brasileiras em sua origem passaram pelo processo de subsidência inicial da fase rift, subsidência térmica (McKenzie, 1978) e ao longo da sua evolução pela subsidência flexural. A modelagem flexural foi calculada na costa do continente Africano (Mohriak e Talwani, 2000) e nas bacias marginais do sudeste brasileiro (Bender et al., 1989), porém foram realizadas em perfis utilizando a aproximação 2-D e rigidez flexural constante.

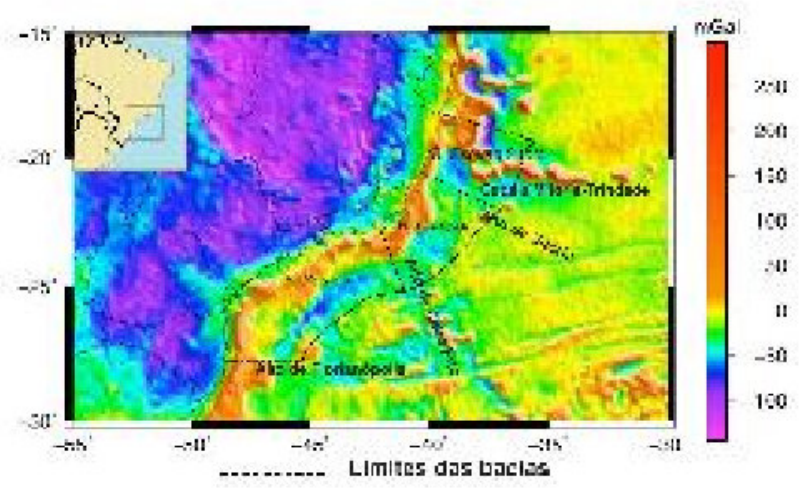

Figura 1 - Mapa de localização das bacias do sudeste do Brasil. Anomalia Bouguer (continente) e Ar-livre (oceano).

Como mostrado na Fig. 1, o segmento SE da margem continental brasileira requer a utilização de uma aproximação 3-D em função do traçado curvilíneo da linha de costa; Neste projeto utilizou-se o método proposto por Sacek (2006) para solução e quantificação da subsidência flexural das bacias marginais brasileiras. Os mapas de isópacas para diferentes fases de sedimentação pós-sal estão disponibilizados em Chang (2003).

Um parâmetro necessário para esta modelagem é a espessura elástica efetiva da litosfera (Te). Esse valor foi calculado para o continente da América do Sul por PérezGussinyé et al. (2007). Observa-se que na região de estudo há um aumento no valor de Te do oceano para o continente. $\mathrm{O}$ valor de Te aumenta com a idade da placa, e é limitado aproximadamente pela isoterma de $450^{\circ} \mathrm{C}$.

\section{Metodologia}

A equação diferencial de quarta ordem que descreve a flexura $\omega=\omega(x, y)$ de uma placa tri-dimensional elástica fina sujeita as cargas verticais q é apresentada na equação 1.

$$
(\mathbf{L} \nabla)^{T} \mathbf{D L} \nabla \omega-q=0
$$


onde,

$$
\begin{gathered}
(\mathbf{L} \nabla)=\left[\frac{\partial^{2}}{\partial x^{2}}, \frac{\partial^{2}}{\partial y^{2}}, 2 \frac{\partial^{2}}{\partial x \partial y}\right]^{T} \\
\mathbf{D}=D\left[\begin{array}{ccc}
1 & v & 0 \\
v & 1 & 0 \\
0 & 0 & (1-v) / 2
\end{array}\right]
\end{gathered}
$$

$D=E T_{e}^{3} / 12\left(1-v^{2}\right)$ é a rigidez da placa, $\mathrm{E}=\mathrm{E}(\mathrm{x}, \mathrm{y})$ é o módulo de Young, $v=v(x, y)$ é o coeficiente de Poisson. $A$ eq. 1 pode ser discretizada usando o método dos elementos finitos (FEM):

$$
\mathbf{K a}=\mathbf{f}
$$

onde,

$$
\begin{gathered}
\mathbf{K a}=\int_{\Omega}\left(\mathbf{B}^{T} \mathbf{D B}+\mathbf{N}^{T} \Delta \rho g \mathbf{N}\right) d \Omega \mathbf{a} \\
\mathbf{f}=\int_{\Omega} \mathbf{N}^{T} \bar{q} d \mathbf{\Omega} \mathbf{a}+\mathbf{f}_{b}
\end{gathered}
$$

onde, $\Omega$ é o domínio estudado, a é um vetor com as incógnitas do problema, $\mathbf{N}$ é uma função que interpola a variável a, $\mathbf{B}=(\mathbf{L} \nabla) \mathbf{N}, \bar{q}$ é a carga e $\Delta \rho . g$ está relacionado com a isostasia.
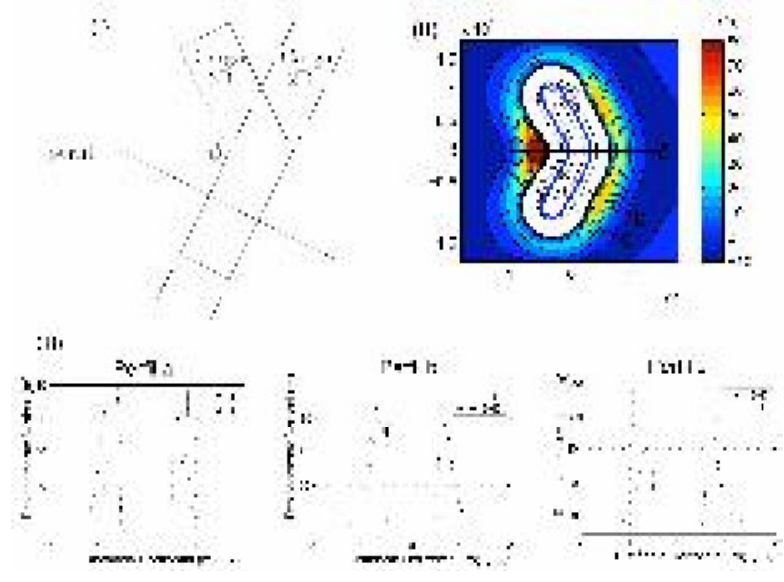

Figura 2 - Modelos sintéticos considerando a geometria da carga 2D ou 3D.(I) Disposição da carga, (II) localização dos perfis e (III) Resultados obtidos do modelo 2D (linha pontilhada) e do modelo 3D (linha traceiada). Modificado de Sacek (2006).

Ao estudar modelos sintéticos para diferentes geometrias de cargas 3-D (figura 2) constatou-se que existem diferenças expressivas nas amplitudes das ombreiras, do que seria observado em modelos 2-D, comprovando a importância da modelagem 3-D.
O tipo de elemento finito utilizado foram os elementos triangulares por sua versatilidade geométrica. Para a geração da malha desses elementos foi utilizado o software DISTMESH desenvolvido por Persson e Strang (2004).

A malha na margem continental foi subdividida em setores, duas faixas no continente e duas faixas no oceano como mostrado na figura 3. Desta forma é possivel levar em conta a variação lateral de Te no espaço e no tempo.

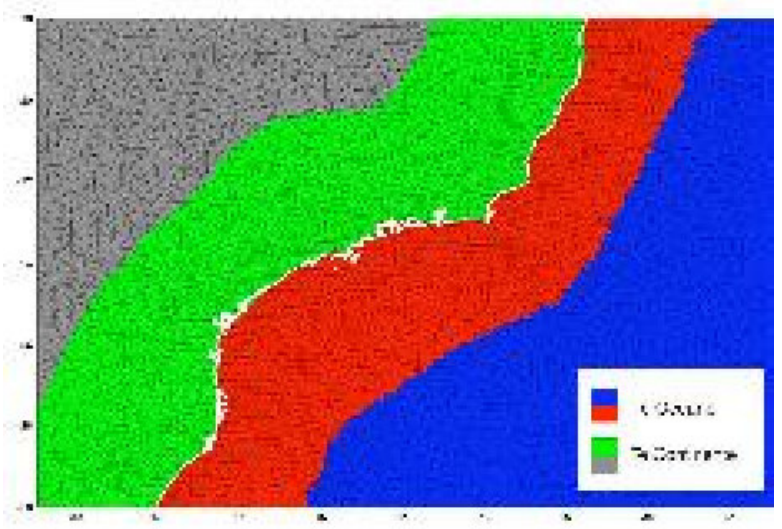

Figura 3 - Malha dos elementos finitos. As faixas em cores indicam as regiões na margem onde valores de Te distintos foram considerados.

Foi realizada a modelagem flexural em 6 períodos de sedimentação como proposto por Chang (2003): Cenomaniano até Coniaciano (99 a $85 \mathrm{Ma}$ ), Santoniano até Campaniano Inferior ( 85 a $70 \mathrm{Ma})$, Campaniano Superior até Maastrichtiano (70 a $65 \mathrm{Ma})$, Paleoceno até Oligoceno Inferior (65 a $28 \mathrm{Ma}$ ), Oligoceno Superior até Mioceno Inferior (28 a $15 \mathrm{Ma}$ ) e Mioceno Superior até o Recente (15 a $0 \mathrm{Ma}$ ).

\section{Resultados}

\begin{tabular}{c|c|c}
\hline Período de Sedimentação & $\begin{array}{c}\text { Intervalo } \\
\text { de tempo } \\
(\mathrm{Ma})\end{array}$ & $\begin{array}{c}\text { Taxa } \\
(\mathrm{m} / \mathrm{Ma})^{*}\end{array}$ \\
\hline $\begin{array}{c}\text { Cenomaniano ao Coniciano } \\
\text { Santoniano ao Campaniano Inferior }\end{array}$ & 14 & 117 \\
Campaniano Superior ao \\
Maastrichtiano & 5 & 101 \\
$\begin{array}{c}\text { Paleoceno ao Oligoceno Inferior } \\
\text { Oligoceno Superior ao Mioceno } \\
\text { Inferior }\end{array}$ & 37 & 48 \\
Mioceno Infeior ao Recente & 13 & 107 \\
\hline
\end{tabular}

Tabela 1 - Valores da taxa de sedimentação para cada período modelado. * $A$ taxa de sedimentação foi definida como espessura máxima do pacote sedimentar dividido pelo intervalo de tempo. 
Os resultados da modelagem flexural estão apresentados na figura 4. As principais observações são:

1. Na fig. 4a é mostrado o resultado da ombreira centrada e com ápice no Arco de Ponta Grossa. O depocentro desta seqüência seria resultante da erosão e retração da escarpa que estendia do sul do Estado de S.Paulo até o sul de Santa Catarina;

2. No Santoniano, tem inicio uma migração para norte dos depocentros deposicionais, e surge um depocentro na bacia de Campos. Um soerguimento incipiente de natureza flexural observado na fase anterior, foi amplificado por um soerguimento térmico no norte do Estado do Rio de Janeiro, cuja erosão forneceu os sedimentos para a margem, que produz um soerguimento flexural importante (Fig. $4 b)$;

3. No período entre $70-65 \mathrm{Ma}$, a máxima deposição volta a ocorrer de forma intensa na bacia de Santos. Em apenas $5 \mathrm{Ma} O$ depocentro desta fase atinge 1 $\mathrm{km}$ de sedimentos depositados (Fig. 4c);

4. No período subseqüente que durou $37 \mathrm{Ma}$, houve baixa taxa deposicional, um novo soerguimento permitiu a deposição de $800 \mathrm{~m}$ na bacia de Campos enquanto que na bacia de Santos a taxa de sedimentação foi muito baixa, e atingiu a máxima espessura de $500 \mathrm{~m}$ na região defronte ao estado do Paraná (Fig. 4d);

5. Com a diminuição da subsidência térmica, uma vez que já havia decorrido mais de $70 \mathrm{Ma}$ desde o fim da fase rift, os depocentros se afastam da linha de costa, a litosfera torna-se cada vez mais rígida, e a ombreira migra para o interior do continente (Figs. 4 e e 4f).

\section{Discussão e Conclusões}

Os resultados preliminares da modelagem flexural das bacias marginais do sudeste do Brasil demonstram a necessidade de se utilizar a aproximação tri-dimensional e levar em conta a variação lateral na rigidez flexural da placa elástica. Demonstra-se também que em dois períodos de sedimentação da bacia de Campos, um soerguimento de natureza termo-magmática deve ter ocorrido que permitiu o fornecimento de sedimentos à bacia. Esses pulsos térmico-magmático podem coincidir com os magmatismos de $80 \mathrm{Ma}$ e $60 \mathrm{Ma}$ ocorrida no sul do cráton S.Francisco e na costa leste do Brasil nesta latitude (Gibson et al., 1995; Thompson et al., 1998; Mohriak et al., 2003). A Fig. 5 mostra a presente distribuição das ombreiras flexurais, resultante das cargas totais pós-sal acumuladas nas bacias marginais. A distribuição dos soerguimentos flexurais explica as feições topográficas e geomorfológicas deste segmento da margem continental do Brasil.

\section{Agradecimentos}

Este trabalho tem o apoio do PRH-19 da ANP com a bolsa de iniciação cientifica a F.L.Pereira, da FAPESP pela bolsa de doutorado a V.Sacek e auxilio à pesquisa a N.Ussami, processo 06/01211-1, e do CNPq processo 300736/2005-2. Agradecemos ao professor Nelsi de Sá por fornecer os dados gravimétricos. Os mapas foram confeccionados no GMT e no Matlab R2008a.

\section{Referências}

Bender, A. A.; Mello, U. T.; Chang H. K., 1989. Reconstituição bidimensional da história geológica de bacias sedimentares - Teoria e uma aplicação na bacia de Campos. Boletim de Geociências da Petrobras, v. 3, n. 1/2:67-85.

Chang, H. K.; Kowsmann, R. O.; Figueiredo, A.M.F.; Bender, A.A., 1992. Tectonics and stratigraphy of the east Brazil rift system: an overview. Tectonophysics, v. 213, n. 1-2:97-138.

Chang, H. K., 2003 - Mapeamento e Interpretação dos Sistemas Petrolíferos da Bacia de Santos. http://www.anp.gov.br/brnd/round5/round5/Apres SemTe c/R5_Santos.pdf, acessado em 25 de junho de 2008.

Gibson, S. A., Thompson, R. N., Leonardos, O. H., Dickin, A. P., Mitchell, J. G., 1995. The Late Cretaceous Impact of the Trindade Mantle Plume: Evidence from Large-volume, Mafic, Potassic Magmatism in SE Brazil J. Petrology, v. 36:189-229.

McKenzie, D., 1978. Some remarks on the development of sedimentary basins. Earth and Planetary Science Letters, v. 10, n.1:25-32.

Mohriak, W. U., Paula, O. B., Szatmari, P., Sobreira, J. F., Parsons, M., Macqueen, J., Undli, T. H., Berstad, S., Weber, M., Horstad, I., 2003. Volcanic provinces in the Eastern Brazilian margin: geophysical models and alternative geodynamic interpretations. In: 8th International Congress of the Brazilian Geophysical Society SBGf.

Pérez-Gussinyé, M., Lowry, A. R., Watts, A. B., 2007. Effective elastic thickness of South America and its implications for intracontinental deformation, Geochemistry Geophysics Geosystems, v. 8, n. 5:1-22.

Persson, P. O. e Strang, G., 2004. A simple mesh generator in matlab, SIAM Review, 46(2):pp 329-345.

Sacek, V. (2006). Modelagem 3D da deformação flexural da litosfera na margem continental do Brasil: Implicações na história de subsidência das bacias marginais. Trabalho de Graduação, Universidade de São Paulo (IAG), pp.41.

Thompson, R. N., Gibson, S. A., Mitchell, J. G., Dickin, A. P., Leonardos, O. H., Brod, J. A., Greenwood, J. C., 1998. Migrating Cretaceous-Eoceno Magmatism in the Serra do Mar Alkaline Province, SE Brazil: Melts from the Deflected Trindade Mantle Plume?. Journal of Petrology, v. 39 , n. 8: 1493-1526.

Watts, A. B.; Ryan, W. B. F., 1976. Flexure of the lithosphere and continental margin basins. Tectonophysics, v. $36: 25-44$. 

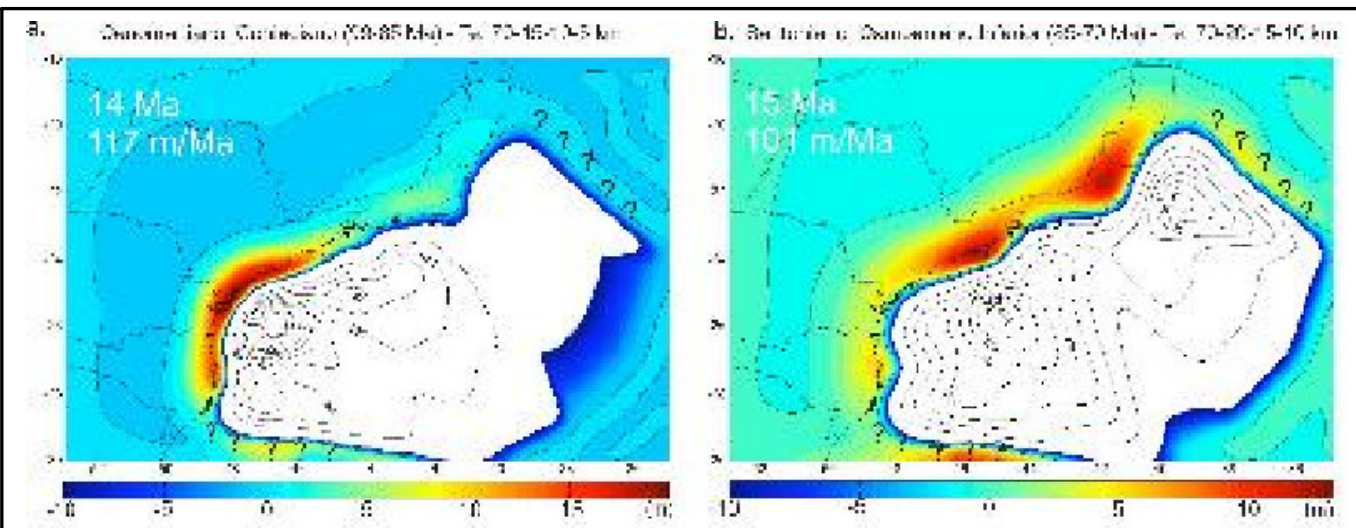

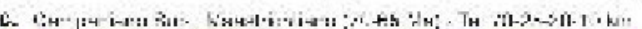

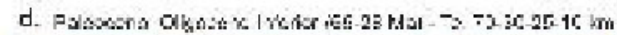
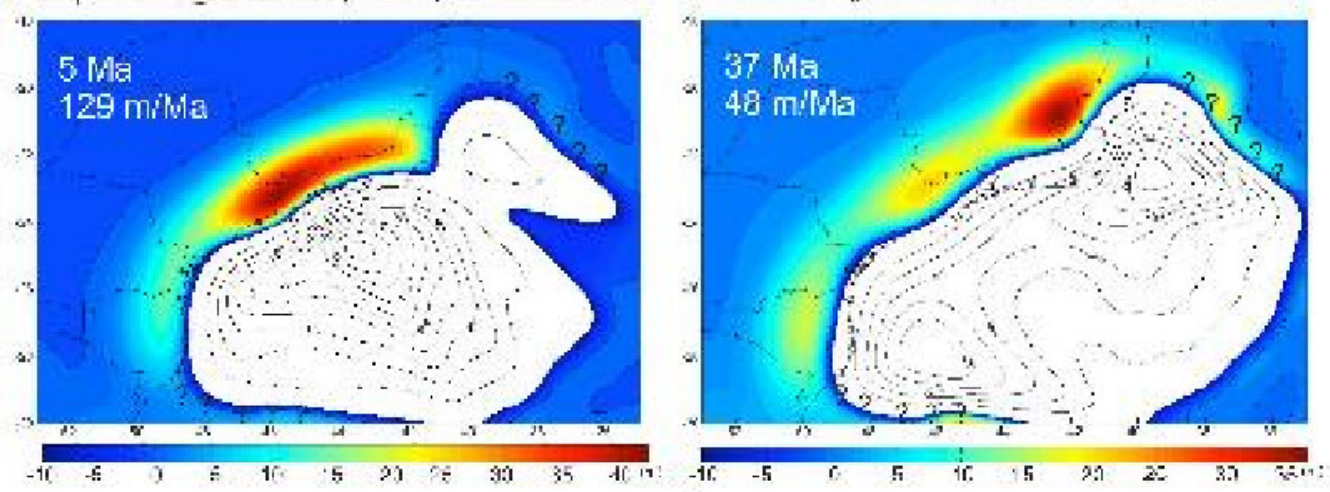

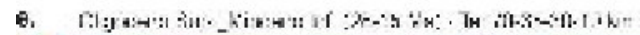
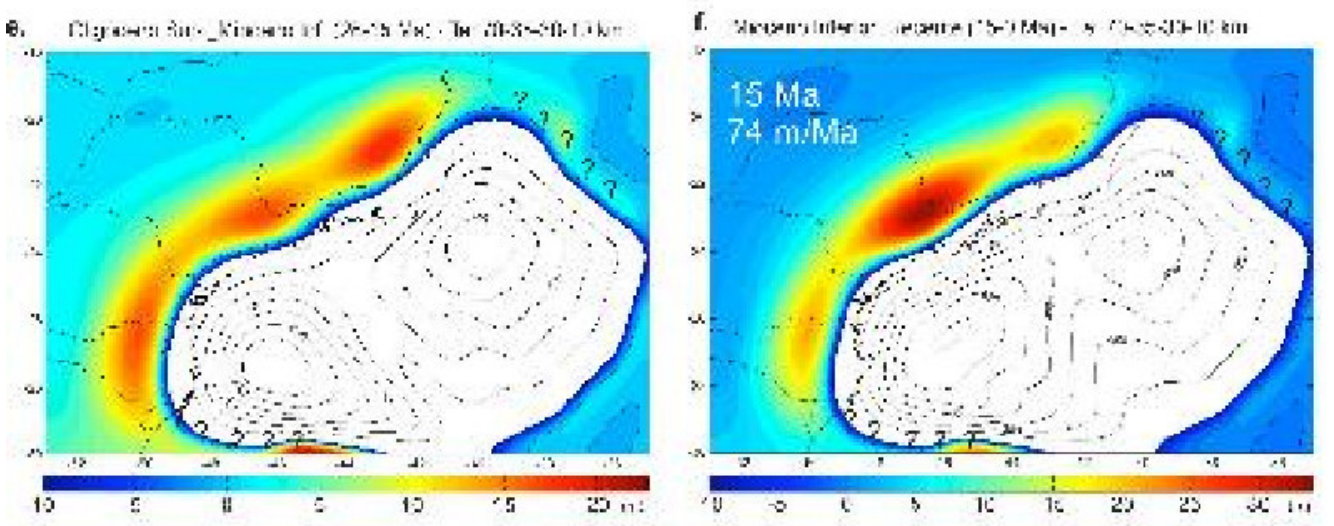

Figura 4 - Modelagem flexural para os seis periodos definidos por Chang (2003). Os valores utilizados para espessura elástica efetiva (Te) são indicados ao lado de cada periodo. Em branco, no interior de cada mapa são indicados o intervalo de tempo e a taxa de sedimentação, conforme definido na Tablea 1. Os pontos de ? nas bordas norte e sul da subsidência indicam a falta de informação sobre as espessuras de sedimento. 


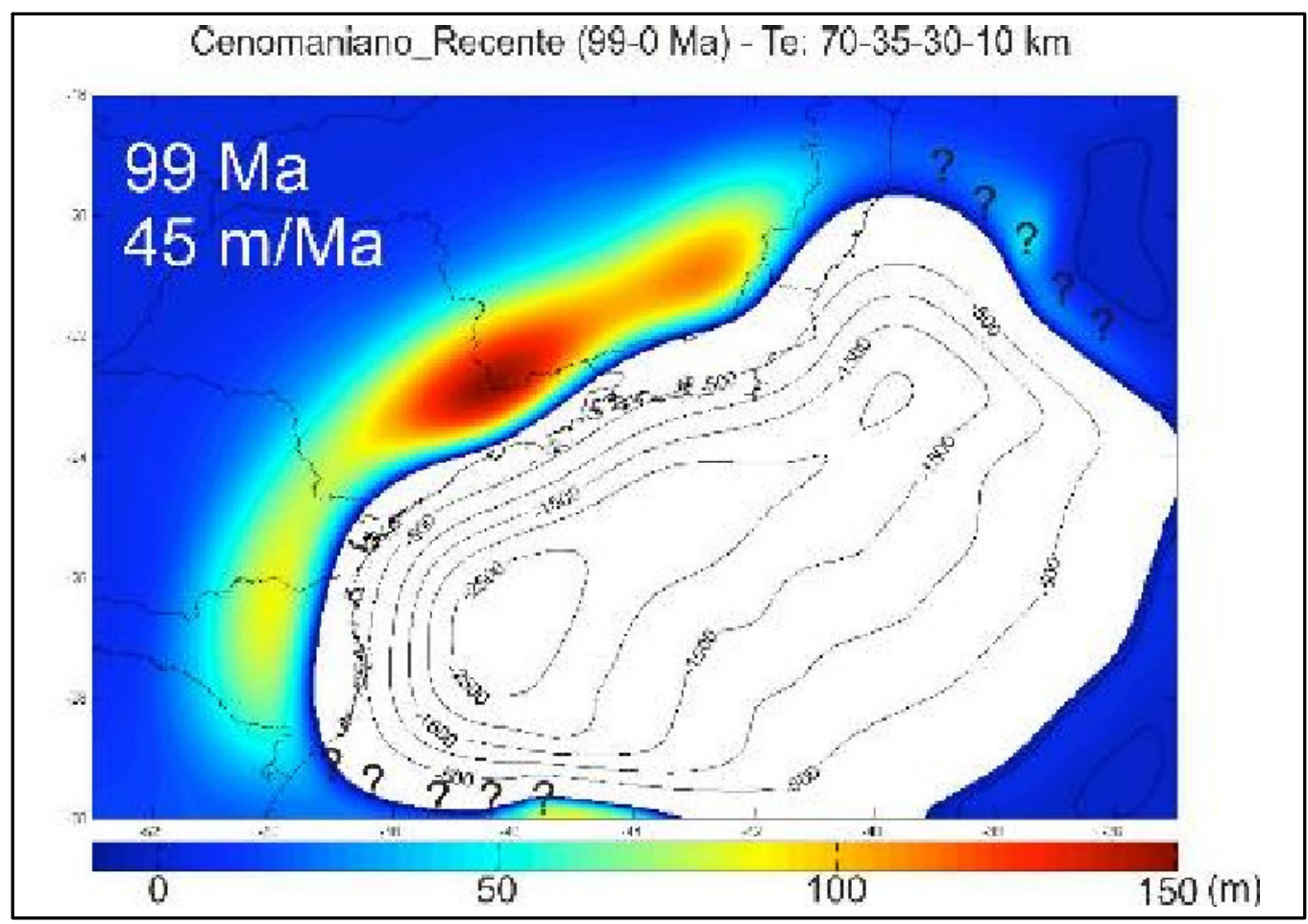

Figura 5 - Modelagem flexural considerando todo o pacote sedimentar pós-sal (estágio atual). Os pontos de ? nas bordas norte e sul da subsidência indicam a falta de informação sobre as espessuras de sedimento. 